\title{
Emerging measurements of atherosclerosis: extra-media thickness, epicardial adipose tissue, and periarterial adipose tissue intima media adventitia index in morbidly obese patients undergoing bariatric surgery
}

\author{
Justyna Domienik-Karłowicz ${ }^{1}$, Wojciech Lisik ${ }^{2}$, Maciej Kosieradzki ${ }^{2}$ Katarzyna Kurnicka ${ }^{1}$, Maciej Haberka ${ }^{3}$, \\ Paweł Ziemiański², Maksymilian Bielecki ${ }^{4}$, Anna Lipińska ${ }^{1}$, Piotr Bienias ${ }^{1}$, Piotr Pruszczyk ${ }^{1}$ \\ ${ }^{1}$ Department of Internal Medicine and Cardiology, Medical University of Warsaw, Warsaw, Poland \\ ${ }^{2}$ Department of General and Transplantation Surgery, Medical University of Warsaw, Warsaw, Poland \\ ${ }^{3}$ Department of Cardiology, School of Health Sciences, Medical University of Silesia, Katowice, Poland \\ ${ }^{4}$ Department of Psychology, SWPS University of Social Sciences and Humanities, Warsaw, Poland
}

Videosurgery Miniinv 2019; 14 (2): 249-254 DOI: https://doi.org/10.5114/wiitm.2019.84678

\begin{abstract}
Introduction: Increased values of emerging fat indices are correlated with increased cardiovascular risk. Aim: To examine the novel non-invasive predictors of coronary disease, namely the carotid extra-media thickness (EMT), PATIMA, and epicardial adipose tissue (EAT), in a group of patients with morbid obesity.

Material and methods: We examined a group of 40 morbidly obese (OB) patients and a control group (CG). All patients were subjected to anthropometric measurements, as well as laboratory and ultrasound examinations.

Results: EATmean and EMTmean differed significantly between groups (OB vs. CG): 5.09 vs. 3.50 and 808.50 vs. 737.00, $p<0.0001$, respectively.

Conclusions: Strong correlations were found between novel non-invasive predictors of coronary disease, namely the carotid extra-media thickness, PATIMA, and epicardial adipose tissue. The above-mentioned fat indices were not found to correlate significantly with BMI or other body weight-related parameters used to assess the adipose tissue content. Further studies are required.
\end{abstract}

Key words: atherosclerosis, bariatric surgery, morbid obesity, extra-media thickness, epicardial adipose tissue.

\section{Introduction}

Obesity is a chronic disease resulting from interactions of multiple genetic, metabolic, environmental, and behavioral factors and leading to increased morbidity and mortality rates [1, 2]. A meta-analysis of 15 prospective studies carried out in a total of 258,000 patients was published in the European Heart Journal to show that abdominal obesity as measured by waist circumference and waist-to-hip ratio (WHR) is associated with increased risk of cardiovascular diseases. A $1-\mathrm{cm}$ increase in waist circumference increases the cardiovascular risk by $2 \%$ while a 0.01 increase in waist-hip ratio (WHR) increases this risk by as much as 5\% [3, 4]. Despite numerous studies, the assessment of cardiovascular risk in the group of patients with morbid obesity undergoing different methods of bariatric surgery requires further verification [5-7]. 


\section{Aim}

Researchers unambiguously state that increased fat indices (EAT, EMT, PATIMA) are correlated with increased cardiovascular risk [6, 8-10]. Thus, we designed a study to examine the novel non-invasive predictors of coronary disease, namely the carotid extra-media thickness (EMT), PATIMA, and epicardial adipose tissue (EAT), as well as their correlation with the conventional cardiovascular risk factors, in a group of patients with morbid obesity undergoing preparation for bariatric surgery. In addition, the study group was compared to an age- and gender-matched control group consisting of subjects with normal body weight.

\section{Material and methods}

\section{Study group}

The study group consisted of 40 female patients with morbid obesity (body mass index (BMI) $>40 \mathrm{~kg} / \mathrm{m}^{2}$ ), average age of $36.4 \pm 9.0$ years; median 37 years (19.0-53.0) not suffering from type 2 diabetes, hospitalized for qualification for elective bariatric surgery. We did not include patients with a history of myocardial infarction, patients with significant valvular defects, chronic renal diseases, chronic obstructive pulmonary disease, active smokers, or patients with severe obstructive sleep apnea $(\mathrm{AHI} \geq 30)$. The control group consisted of 15 nonobese females at the mean age of $36 \pm 8.34$ years, with average body weight of $60.72 \pm 5.12 \mathrm{~kg}$. Patients in the control group were selected so as to match the age and gender distribution in the study group.

\section{Anthropometric examinations and laboratory investigations}

All patients were subjected to body weight, BMI, body fat weigh and lean body mass measurements using a TBF-300 Tanita Body Composition Analyzer. The device automatically analyzes body composition with the accuracy of $\pm 0.1 \mathrm{~kg}$ on the basis of differences in electric bioimpedance, i.e. the differences in electric conductivity between aqueous and adipose compartments. In addition, the body surface area (BSA) was determined using the Mosteller equation: (height ${ }^{0.5}$ $\times$ body weight ${ }^{0.5} / 60$, and excess body weight (EBW) was calculated using the current body weight minus ideal body weight formula. The ideal body weight was calculated as height in $\mathrm{cm}-100-10 \%$.
Laboratory investigations were carried out including fasting glucose, 75-g oral glucose tolerance test, fasting insulin, glycated hemoglobin $\left(\mathrm{HbA}_{1 \mathrm{c}}\right)$, lipid profile, glomerular filtration rate (GFR), creatinine, serum glucose $(\mathrm{mg} / \mathrm{dl})$ and insulin levels ( $\mu \mathrm{IU} /$ $\mathrm{ml}$ ) as measured by immunochromatography. Normal insulin level was defined as 5-15 $\mu \mathrm{IU} / \mathrm{ml}$. In addition, the HOMA insulin resistance and quantitative insulin sensitivity check indices (QUICKI) were calculated using the formulas HOMA-IR $=$ insulin $(\mu \mathrm{IU} / \mathrm{ml})$ $\times$ glucose $(\mathrm{mmol} / \mathrm{l}) / 22.5$ and QUICKI $=1 /(\log ($ insulin $(\mu \mathrm{IU} / \mathrm{ml}))+\log ($ glucose $(\mathrm{mmol} / \mathrm{l})))$.

\section{Ultrasound scans}

The following measurements were made: EMT is the distance between the carotid media-adventitia border and the jugular lumen interface. The measurements were made manually due to the lack of dedicated software. The average of 3 measurements was used for calculations. We used a standardized EMT protocol, including measurements along a 7-mm segment starting $3 \mathrm{~mm}$ proximally to the carotid bulb. All examinations were performed and evaluated by a single experienced physician. Examinations were performed using a Philips iE 33 system (Andover, Massachusetts USA) with a 5-7 MHz linear array transducer.

The EAT measurements were carried out on the free right ventricular wall as visualized in two projections, the long axis and short axis parasternal view; the average of 3 measurements was used for calculations. This is a standardized measurement method correlated with the gold standard of MRI measurements. All examinations were performed and evaluated by a single physician. Examinations were carried out using a Philips iE 33 system (Andover, Massachusetts USA) with a $2.5-3.5 \mathrm{MHz}$ transducer.

The periarterial adipose tissue intima media adventitia (PATIMA) index was calculated using the formula PATIMA $=(E M T / B M I \times 35)+$ IMT + EAT $\times$ 60. EMT and EAT measurements were carried out as described above. The IMT, i.e. the thickness of the carotid artery tunica intima and tunica media, was measured in fasting subjects as an average of 3 measurements of the posterior walls of the left and right common carotid arteries $2 \mathrm{~cm}$ from the bulb. All examinations were performed and evaluated by a single physician. Examinations were carried 
out using a Philips iE 33 system (Andover, Massachusetts USA) with a 5-7 MHz linear array transducer.

The study was approved by the appropriate bioethics committee.

\section{Statistical analysis}

Descriptive statistics are reported as medians with range and means with standard deviations. Due to the relatively small size of the control group, the Mann-Whitney test was used in all comparisons of continuous variables. Relationships between continuous variables in the patient group were estimated using Spearman's rho coefficient. The level of statistical significance was established at $p<0.05$. Calculations were performed using the SAS 9.2 software.

\section{Results}

\section{Clinical characteristics}

Arterial hypertension $(\mathrm{H})$ was defined on the basis of arterial blood pressure measurements (systolic blood pressure $\geq 140 \mathrm{~mm} \mathrm{Hg}$ and/or diastolic blood pressure $\geq 90 \mathrm{~mm} \mathrm{Hg}$ ) or of the anti-hypertensive treatment received at the time of the study. On the basis of these criteria, $\mathrm{H}$ was diagnosed in $95 \%$ of patients, including 30 patients treated with at least two anti-hypertensive agents (including angiotensin-converting enzyme inhibitors and diuretics) and 8 patients treated with angiotensin-converting enzyme inhibitors alone.

Prediabetic condition was diagnosed on the basis of impaired fasting glycemia (IFG) or impaired glucose tolerance (IGT). IFG was detected in $6(15 \%)$ patients while IGT was detected in 7 (17.5\%) patients; in 2 patients both disorders were observed simultaneously. In 19 (48.71\%) patients fasting insulin levels were found to exceed $15 \mu \mathrm{IU} / \mathrm{ml}$, indicating hyperinsulinemia. In 26 (67\%) patients, HOMA-IR exceeded the normal limit of 2.5. Lipid metabolism disorders were diagnosed in $22(55 \%)$ patients while $8(20 \%)$ patients suffered from mild to moderate obstructive sleep apnea. All patients were assessed for the possible occurrence of metabolic syndrome. All patients met the NCEP-ATP III diagnostic criteria of metabolic syndrome as defined in 2001 and subsequent updates. Also, all patients met the diagnostic criteria as defined by the IDF in 2005.

Basic characteristics of patients are presented in Table I. Fat tissue parameters are presented in Table II.
The comparison of fat tissue parameters between patients with morbid obesity and patients in the control group is presented in Table III.

EATmean differs significantly between groups (OB vs. CG): 5.09 vs. $3.50, p<0.0001$. Moreover, EMTmean differs significantly between groups (OB vs. (G): 808.50 vs. $737.00, p<0.0001$.

Correlations between selected parameters within the study group are presented in Table IV.

\section{Discussion}

Our study facilitated noninvasive evaluation of early atherosclerotic markers in patients with morbid obesity as compared to a control group; several important findings were made. When comparing both the age- and gender-matched groups, note should be taken of the statistically significant differences in

Table I. Basic characteristics of patients with morbid obesity

\begin{tabular}{|c|c|c|}
\hline Parameter & Mean & SD \\
\hline Body weight [kg] & 132.03 & 18.42 \\
\hline $\mathrm{BMI}\left[\mathrm{kg} / \mathrm{m}^{2}\right]$ & 47.73 & 6.18 \\
\hline FAT [\%] & 49.15 & 3.83 \\
\hline FFM [kg] & 66.78 & 7.84 \\
\hline BSA $M\left[m^{2}\right]$ & 2.47 & 0.20 \\
\hline EBW $[k g]$ & 72.34 & 16.75 \\
\hline Fasting glucose [mg/dl] & 90.4 & 10.19 \\
\hline Fasting insulin $[\mu \mathrm{l} \mathrm{U} / \mathrm{ml}]$ & 17.68 & 11.29 \\
\hline 2 h glucose $[\mathrm{mg} / \mathrm{dl}]$ & 109.58 & 31.16 \\
\hline $2 \mathrm{~h}$ insulin $[\mu \mathrm{lU} / \mathrm{ml}]$ & 60.72 & 53.93 \\
\hline QUICKI & 0.94 & 0.19 \\
\hline HOMA-IR & 3.89 & 2.33 \\
\hline Creatinine [mg/dl] & 0.76 & 0.16 \\
\hline Total cholesterol [mg/dl] & 199.15 & 35.13 \\
\hline HDL-chol [mg/dl] & 52.55 & 10.97 \\
\hline LDL-chol [mg/dl] & 120.3 & 31.59 \\
\hline Triglycerides [mg/dl] & 124.78 & 47.7 \\
\hline $\mathrm{hsCRP}[\mathrm{mg} / \mathrm{l}]$ & 10.19 & 6.92 \\
\hline $\mathrm{HbA}_{1 \mathrm{c}}(\%)$ & 5.8 & 0.89 \\
\hline $\mathrm{GFR}[\mathrm{ml} / \mathrm{min}]$ & 96.16 & 20.76 \\
\hline
\end{tabular}


Table II. Fat tissue parameters in patients with morbid obesity

\begin{tabular}{|lccccc|}
\hline Parameter & Min. & Med. & Mean & Max. & SD \\
\hline EAT_LAX & 3.50 & 5.29 & 5.27 & 7.63 & 0.83 \\
\hline EAT_SAX & 3.70 & 5.11 & 5.37 & 7.46 & 7.01 \\
\hline EAT_MEAN & 3.60 & 5.09 & 5.32 & 1771.50 & 0.89 \\
\hline PATIMA & 1212.53 & 1497.32 & 1507.90 & 1788.90 & 124.78 \\
\hline PATIMA_LAX & 1221.53 & 1495.57 & 1504.78 & 888.00 & 39.67 \\
\hline EMT_MEAN & 721.00 & 808.50 & 804.73 & 0.16 & 0.02 \\
\hline EAT_BMI & 0.07 & 0.11 & 0.11 & 20.97 & 2.13 \\
\hline EMT_BMI & 12.26 & 17.06 & 17.10 & & \\
\hline
\end{tabular}

Table III. Comparison of fat tissue parameters between patients with morbid obesity (OB) and patients in the control group (CG)

\begin{tabular}{|lccccccc|}
\hline Parameter & GK min. & GK median & GK max. & OB min. & OB median & OB max. & $P$-value \\
\hline EAT LAX & 3.20 & 3.56 & 3.88 & 3.50 & 5.29 & 7.63 & $<0.0001$ \\
\hline EAT SAX & 3.10 & 3.42 & 3.90 & 3.70 & 5.11 & 7.46 & $<0.0001$ \\
\hline EATmean & 3.31 & 3.50 & 3.72 & 3.60 & 5.09 & 7.34 & $<0.0001$ \\
\hline PATIMA & 1723.69 & 1835.48 & 1970.81 & 1212.53 & 1497.32 & 1771.50 & $<0.0001$ \\
\hline PATIMA LAX & 1722.49 & 1844.53 & 1977.11 & 1221.53 & 1495.57 & 1788.90 & $<0.0001$ \\
\hline EMTmean & 703.00 & 737.00 & 788.00 & 721.00 & 808.50 & 888.00 & $<0.0001$ \\
\hline EAT BMI & 0.14 & 0.16 & 0.18 & 0.07 & 0.11 & 0.16 & $<0.0001$ \\
\hline EMT BMI & 31.75 & 33.96 & 38.89 & 12.26 & 17.06 & 20.97 & $<0.0001$ \\
\hline BMI & 18.90 & 21.90 & 23.80 & 40.10 & 46.73 & 64.25 & $<0.0001$ \\
\hline FAT \% & 18.30 & 24.50 & 36.30 & 39.60 & 49.65 & 56.10 & $<0.0001$ \\
\hline FM & 9.20 & 14.80 & 23.30 & 47.17 & 63.43 & 96.94 & $<0.0001$ \\
\hline FFM & 39.70 & 45.80 & 50.70 & 53.80 & 67.27 & 83.38 & $<0.0001$ \\
\hline
\end{tabular}

adipose tissue indices. This is important since EMT, EAT, and PATIMA were documented to be correlated with the incidence and stage of coronary heart disease $[9,11]$. This was the first study conducted in a group of patients with morbid obesity and indicative of the presence of correlations between the novel adipose tissue markers and the risk of cardiovascular diseases.

Based on the study by Haberka et al., the mean EMT values in the group of patients with morbid obesity warrant the assumption that the coronary heart disease encompasses 1 coronary vessel in $\geq 50 \%$ of patients. In this group of patients, the sensitivity and specificity values for EMT are $60 \%$ and $76 \%$, respec- tively. The pioneering character of the study requires that these results be validated in a larger group of patients, perhaps in reference to calcium scores as obtained in coronary CT scans or to the coronary CT scans in patients in whom the risk was determined to be the highest. Of note, the results of our study should be validated in a larger group of patients and in patients with coronary heart disease confirmed in an invasive manner.

Moreover, epicardial adipose tissue (EAT) was shown to present with characteristics similar to those of the abdominal adipose tissue. It may cause local inflammation and thus promote coronary atherosclerosis [12]. Moreover, it is now evident that 


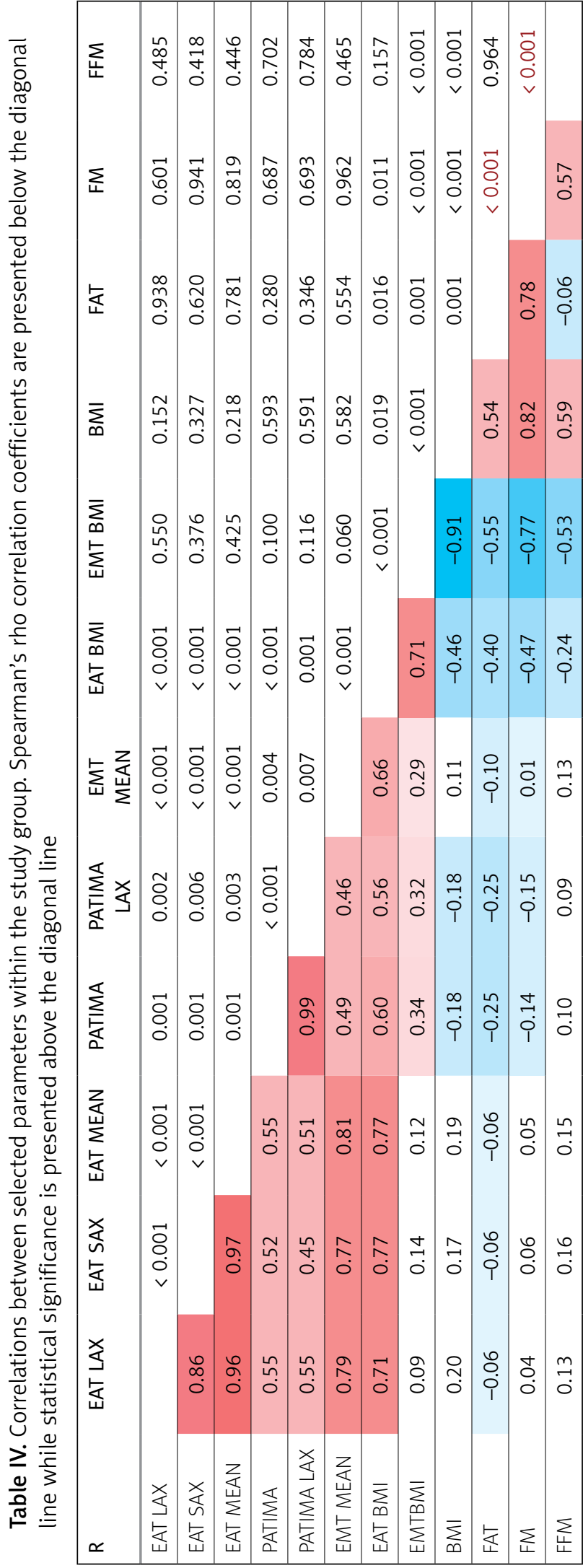

EAT-secreted bioactive molecules may play an important role in the pathogenesis of coronary artery disease and cardiac arrhythmia [13-15]. Of note, lower EAT density and increased EAT volume were associated with coronary calcification, serum levels of plaque inflammatory markers and MACE. That is why it could be a good tool for screening patients undergoing bariatric surgery, facilitating diagnosis of patients who need further treatment $[16,17]$. Moreover, in a meta-analysis increasing EAT was associated with the presence of high-risk plaque. However, also this meta-analysis underlines that further evaluation is needed [18].

\section{Clinical implications}

Yerramasu et al. showed that patients with increased EAT are at higher risk of quick atherosclerotic plaque growth. In the Framingham study, adipose tissue measurements were correlated with higher incidence of myocardial infarction. After elimination of limitations such as the population size and gender bias, our study may contribute to these adipose tissue parameters being used in cardiovascular risk screening examinations $[19,20]$. Secondly, the number of patients included in our group was relatively small. Moreover, the lack of a long follow-up may be considered as a limitation, so further long-term studies are needed.

\section{Conclusions}

Strong correlations were identified between EAT PATIMA, and EMT MEAN. The above-mentioned fat indices were not found to correlate significantly with $\mathrm{BMI}$ or other body weight-related parameters used to assess the adipose tissue content. Further studies are required to determine whether these markers may be used for cardiovascular risk screening in this group of patients.

\section{Conflict of interest}

The authors declare no conflict of interest.

\section{References}

1. Dicker D, Atar E, Kornowski R, Bachar GN. Increased epicardial adipose tissue thickness as a predictor for hypertension: a cross-sectional observational study. J Clin Hypertens 2013; 15: 893-8

2. Fried M, Yumuk V, Oppert JM, et al. Interdisciplinary European guidelines on metabolic and bariatric surgery. Obes Surg 2014; 24: 42-55. 
3. de Koning L, Merchant AT, Pogue J, Anand SS. Waist circumference and waist-to-hip ratio as predictors of cardiovascular events: meta-regression analysis of prospective studies. Eur Heart J 2007; 28: 850-6.

4. Kostecka M, Bojanowska M. Problems in bariatric patient care challenges for dieticians. Videosurgery Miniinv 2017; 12: 207-15.

5. Domienik-Karlowicz J, Dzikowska-Diduch O, Lisik W, et al. Short-term cardiometabolic risk reduction after bariatric surgery. Hellen J Cardiol 2015; 56: 61-5.

6. Haberka M, Lelek M, Bochenek T, et al. Novel combined index of cardiometabolic risk related to periarterial fat improves the clinical prediction for coronary artery disease complexity. Atherosclerosis 2018; 268: 76-83.

7. Frask A, Orlowski M, Dowgiallo-Wnukiewicz N, et al. Clinical evaluation of $\mathrm{C}$-reactive protein and procalcitonin for the early detection of postoperative complications after laparoscopic sleeve gastrectomy. Videosurgery Miniinv 2017; 12: 160-5.

8. Haberka M, Okopien B, Gasior Z. Obesity, ultrasound indexes of fat depots and lipid goal attainment in patients with high and very high cardiovascular risk: a novel approach towards better risk reduction. Nutr Metab Cardiovasc Dis 2016; 26: 123-33.

9. Haberka M, Gasior Z. A carotid extra-media thickness, PATIMA combined index and coronary artery disease: comparison with well-established indexes of carotid artery and fat depots. Atherosclerosis 2015; 243: 307-13.

10. Wu FZ, Chou KJ, Huang YL, Wu MT. The relation of location-specific epicardial adipose tissue thickness and obstructive coronary artery disease: systemic review and meta-analysis of observational studies. BMC Cardiovasc Disord 2014; 14: 62.

11. von Gise A, Pu WT. Endocardial and epicardial epithelial to mes enchymal transitions in heart development and disease. Circ Res 2012; 110: 1628-45.

12. Aune D, Schlesinger S, Norat T, Riboli E. Body mass index, abdominal fatness, and the risk of sudden cardiac death: a systematic review and dose-response meta-analysis of prospective studies. Eur J Epidemiol 2018; 33: 711-22.

13. Guglielmi V, Sbraccia P. Epicardial adipose tissue: at the heart of the obesity complications. Acta Diabetol 2017; 54: 805-12.

14. Patel VB, Shah S, Verma S, Oudit GY. Epicardial adipose tissue as a metabolic transducer: role in heart failure and coronary artery disease. Heart Fail Rev 2017; 22: 889-902.

15. Goeller M, Achenbach S, Marwan M, et al. Epicardial adipose tissue density and volume are related to subclinical atherosclerosis, inflammation and major adverse cardiac events in asymptomatic subjects. J Cardiovasc Comput Tomogr 2018; 12: 67-73.

16. Csige I, Ujvarosy D, Szabo Z, et al. The Impact of obesity on the cardiovascular system. J Diabetes Res 2018; 2018: 3407306.

17. Jonker FHW, van Houten VAA, Wijngaarden LH, et al. Age-related effects of bariatric surgery on early atherosclerosis and cardiovascular risk reduction. Obes Surg 2018; 28: 1040-6.

18. Nerlekar N, Brown AJ, Muthalaly RG, et al. Association of epicardial adipose tissue and high-risk plaque characteristics: a systematic review and meta-analysis. J Am Heart Assoc 2017; 6: pii: e006379.

19. Yerramasu A, Dey D, Venuraju S, et al. Increased volume of epicardial fat is an independent risk factor for accelerated pro- gression of sub-clinical coronary atherosclerosis. Atherosclerosis 2012; 220: 223-30.

20. Mahabadi AA, Lehmann N, Kalsch $\mathrm{H}$, et al. Association of epicardial adipose tissue with progression of coronary artery calcification is more pronounced in the early phase of atherosclerosis: results from the Heinz Nixdorf recall study. JACC Cardiovasc Imaging 2014; 7: 909-16.

Received: 30.01.2019, accepted: 3.04.2019. 